\title{
High order optimal control of space trajectories with uncertain boundary conditions
}

\author{
P. Di Lizia*, R. Armellin, F. Bernelli-Zazzera \\ Politecnico di Milano, Dipartimento di Ingegneria Aerospaziale, Via La Masa 34, 20156 \\ Milano, Italy \\ M. Berz \\ Department of Physics and Astronomy, Michigan State University, East Lansing, MI, \\ $U S A$
}

\begin{abstract}
A high order optimal control strategy is proposed in this work, based on the use of differential algebraic techniques. In the frame of orbital mechanics, differential algebra allows to represent, by high order Taylor polynomials, the dependency of the spacecraft state on initial conditions and environmental parameters. The resulting polynomials can be manipulated to obtain the high order expansion of the solution of two-point boundary value problems. Since the optimal control problem can be reduced to a two-point boundary value problem, differential algebra is used to compute the high order expansion of the solution of the optimal control problem about a reference trajectory. Whenever perturbations in the nominal conditions occur, new optimal control laws for perturbed initial and final states are obtained by the mere evaluation of polynomials. The performances of the method are assessed on lunar landing, rendezvous maneuvers, and a low-thrust Earth-Mars
\end{abstract}

${ }^{*}$ Corresponding author. E-mail: pierluigi.dilizia@polimi.it 
transfer.

Keywords: Optimal control, Space trajectories, High-order methods, Differential algebra, Uncertain boundary conditions

\section{Introduction}

Nominal space trajectories are usually designed by solving optimal control problems that minimize the control action to meet mission constraints. However, uncertainties and disturbances affect the spacecraft dynamics in real scenarios. Moreover, state identification is influenced by navigation errors; consequently, the spacecraft state is only known with a given accuracy. Thus, after the nominal solution is computed, an optimal feedback control strategy that assures the satisfaction of mission constraints must be implemented. More specifically, given an initial deviation of the spacecraft state from its nominal value or a perturbation on the nominal final target conditions, the optimal control aims at canceling the effects of such errors by correcting the nominal control law, while minimizing propellant consumption.

Optimal feedback control was originally developed for linear systems. In linear optimal control theory, the system is assumed linear and the feedback controller is constrained to be linear with respect to its input [1]. The technological challenges imposed by the recent advances in aerospace engineering are demanding stringent accuracy requirements and cost reduction for the control of nonlinear systems. Unfortunately, the accuracy of linearized dynamics can drop off rapidly in nonlinear aerospace applications, affecting the performances of linear optimal controller. Thus, nonlinear optimal feedback 
control theory has gained interest in the past decades.

Various aspects of nonlinear optimal control have been addressed. Several techniques are available for solving control-affine problems, which are mainly based on dynamic programming or calculus of variations. In Bellmans dynamic programming, the problem is approached by reducing it to solving the nonlinear first-order partial differential Hamilton-Jacobi-Bellman (HJB) equation [2]. The solution to the HJB equation determines the optimal feedback control, but its use is very intricate in practical problems. An alternative approach is based on the calculus of variations and Pontryagins maximum principle, which show the Hamiltonian nature of the second order information of the optimal control problem [3]. Within this frame, the optimal control problem is reduced to a two-point boundary value problem (TPBVP) that is solved, in general, by successive approximation of the optimal control input using iterative numerical techniques. However, the solution determined is only valid for one set of boundary conditions, which prevents its immediate use for feedback control.

The complexity of finding the exact solution of the HJB equation has motivated research for approximated methods that are able to supply suboptimal laws for the control of nonlinear systems about reference solutions. In Bryson and Ho [2], an approximating technique is presented, based on a second order expansion of the augmented performance index of the optimal control problem, which is referred to as neighboring extremal paths computation. The State-dependent Riccati equation (SDRE) control method is among the more attractive tools to obtain such approximate solutions. It was originally proposed by Pearson [4], and Wernli and Cook [5], and then 
described in details by Mracek and Cloutier [6], and Beeler [7]. This method involves manipulating the governing dynamic equations into a pseudo-linear non-unique form in which system matrices are given as a function of the current state and minimizing a quadratic-like performance index. An algebraic Riccati equation using the system matrices is then solved repetitivily online to give the optimal control law. Thus, the SDRE approach might turn out to be computationally expensive when the solution of the Riccati equation is not properly managed. This can prevent its use for real-time optimal control. A significant computational advantage can be obtained with the $\theta-D$ technique [8]. Similarly to $\operatorname{SDRE}$, the $\theta-D$ technique relies on an approximate solution to the HJB equation. However, it offers a great computational advantage for onboard implementation without solving the Riccati equation repetitively at every instant.

Recent advances have been made in the frame of variational approach to optimal control theory. Second order methods were introduced by Bullock [9] and then extended by Olympio [10] to space trajectory design. Based on the Hamiltonian nature of the optimal control problem, the method compute a linear control update iteratively using the gradient of the Hamiltonian function. A higher order approach was introduced by Park and Scheeres [11] through the theory of canonical transformations. More specifically, canonical transformations solve boundary value problems between Hamiltonian coordinates and momenta for a single flow field. Thus, based on the reduction of the optimal control problem to an equivalent boundary value problem, they can be effectively used to solve the optimal control problem analytically as a function of the boundary conditions, which is instrumental to optimal feed- 
back control. The main difficulty of this approach is finding the generating functions via the solution of the Hamilton-Jacobi equation. This problem was solved by Park and Scheeres by expanding the generating function in power series of its arguments.

Differential algebraic (DA) techniques [12] are used in this work to develop an alternative approach to the generating function method. Differential algebra serves the purpose of computing the derivatives of functions in a computer environment. More specifically, by substituting the classical implementation of real algebra with the implementation of a new algebra of Taylor polynomials, it expands any function $f$ of $v$ variables into its Taylor series up to an arbitrary order $n$. DA techniques are used in this work to represent the dependency of the spacecraft state on the initial conditions by means of high order Taylor polynomials. Then, the resulting Taylor polynomials are manipulated to impose the boundary and optimality conditions of the optimal control problem. This enables the expansion of the solution of the optimal control problem with respect to the initial conditions about an available reference trajectory. The resulting Taylor polynomials can be evaluated for new solutions of the optimal control problem, so avoiding repetitive runs of classical iterative procedures.

The paper is organized as follows. A brief introduction to differential algebra is given in Sect. 2. Being at the basis of the proposed methods, the possibility of expanding the flow of ODEs is presented in Sect. 3. The optimal control problem and the algorithm for the high order expansion of its solution are illustrated in Sect. 4 and Sect. 5, respectively. The application of the algorithm to a rendezvous maneuver, a lunar landing, and a low-thrust 
Earth-Mars transfer problem is addressed in Sect. 6.

\section{Differential Algebra}

DA techniques find their origin in the attempt to solve analytical problems by an algebraic approach [12]. Historically, the treatment of functions in numerics has been based on the treatment of numbers, and the classical numerical algorithms are based on the mere evaluation of functions at specific points. DA techniques are based on the observation that it is possible to extract more information on a function rather than its mere values. The basic idea is to bring the treatment of functions and the operations on them to the computer environment in a similar way as the treatment of real numbers. Referring to Fig. 1, consider two real numbers $a$ and $b$. Their transformation into the floating point representation, $\bar{a}$ and $\bar{b}$ respectively, is performed to operate on them in a computer environment. Then, given any operation $\times$ in the set of real numbers, an adjoint operation $\otimes$ is defined in the set of FP numbers such that the diagram in figure commutes. (The diagram commutes approximately in practice, due to truncation errors.) Consequently, transforming the real numbers $a$ and $b$ in their FP representation and operating on them in the set of FP numbers returns the same result as carrying out the operation in the set of real numbers and then transforming the achieved result in its FP representation. In a similar way, suppose two sufficiently regular functions $f$ and $g$ are given. In the framework of differential algebra, the computer operates on them using their Taylor series expansions, $F$ and $G$ respectively. Therefore, the transformation of real numbers in their FP representation is now substituted by the extraction of the Taylor expansions 

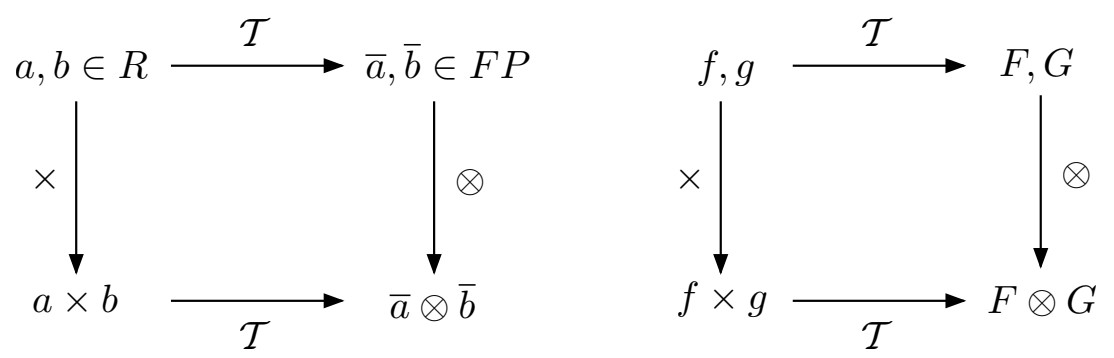

Figure 1: Analogy between the floating point representation of real numbers in a computer environment (left figure) and the introduction of the algebra of Taylor polynomials in the differential algebraic framework (right figure).

of $f$ and $g$. For each operation in the function space, an adjoint operation in the space of Taylor polynomials is defined such that the corresponding diagram commutes. Extracting the Taylor expansions of $f$ and $g$ and operating on them in the function space returns the same result as operating on $f$ and $g$ in the original space and then computing the Taylor expansion of the resulting function. The straightforward implementation of differential algebra in a computer allows to compute the Taylor coefficients of a function up to a specified order $n$, along with the function evaluation, with a fixed amount of effort. The Taylor coefficients of order $n$ for sums and product of functions, as well as scalar products with reals, can be computed from those of summands and factors; therefore, the set of equivalence classes of functions can be endowed with well-defined operations, leading to the so-called truncated power series algebra [13].

Similarly to the algorithms for floating point arithmetic, the algorithm for functions followed, including methods to perform composition of functions, to invert them, to solve nonlinear systems explicitly, and to treat common elementary functions [12]. In addition to these algebraic operations, also 
the analytic operations of differentiation and integration are introduced, so finalizing the definition of the DA structure. The differential algebra sketched in this section was implemented by Berz and Makino in the software COSYInfinity [14].

\section{High Order Expansion of ODE Flow}

The differential algebra introduced in the previous section allows to compute the derivatives of any function $f$ of $v$ variables up to an arbitrary order $n$, along with the function evaluation. This has an important consequence when the numerical integration of an ODE is performed by means of an arbitrary integration scheme. Any explicit integration scheme is based on algebraic operations, involving the evaluations of the ODE right hand side at several integration points. Therefore, carrying out all the evaluations in the DA framework allows differential algebra to compute the arbitrary order expansion of the flow of a general ODE initial value problem.

Without loss of generality, consider the scalar initial value problem

$$
\left\{\begin{array}{l}
\dot{x}=f(x) \\
x\left(t_{i}\right)=x_{i} .
\end{array}\right.
$$

Replace the point initial condition $x_{i}$ with its DA representative $\left[x_{i}\right]$, i.e., consider the variation $\left[x_{i}\right]=x_{i}^{0}+\delta x_{i}$, where $x_{i}^{0}$ is the reference point for the expansion. If all the operations of the numerical integration scheme are carried out in the framework of differential algebra, the Taylor expansion of the solution with respect to the initial condition is obtained at each step. As an example, consider the forward Euler scheme

$$
x_{k}=x_{k-1}+\Delta t \cdot f\left(x_{k-1}\right)
$$


and analyze the first integration step; i.e.,

$$
x_{1}=x_{0}+\Delta t \cdot f\left(x_{0}\right)
$$

where $x_{0}=x_{i}$. Substitute the initial value with $\left[x_{0}\right]=\left[x_{i}\right]=x_{i}^{0}+\delta x_{i}$ in Eq. (3) for

$$
\left[x_{1}\right]=\left[x_{0}\right]+\Delta t \cdot f\left(\left[x_{0}\right]\right) .
$$

If the function $f$ is evaluated in the DA framework, the output of the first step, $\left[x_{1}\right]$, is the Taylor expansion of the solution $x_{1}$ at $t_{1}$ with respect to the initial condition about the reference point $x_{i}^{0}$. The previous procedure can be repeated for the subsequent steps until the last integration step is reached. The result at the final step is the $n$-th order Taylor expansion of the flow of the initial value problem (1) at the final time $t_{f}$. Thus, the expansion of the flow of a dynamical system can be computed up to order $n$ with a fixed amount of effort.

\section{Optimal Control Problem}

Suppose the spacecraft moves under the general dynamics

$$
\dot{\boldsymbol{x}}=\boldsymbol{f}(\boldsymbol{x}(t), \boldsymbol{u}(t), t),
$$

where $\boldsymbol{x}=\left\{x_{1}, \ldots, x_{v}\right\}$ is the state vector and $\boldsymbol{u}=\left\{x_{1}, \ldots, x_{m}\right\}$ is the control vector $(m \leq v)$. The optimal control problem aims at finding the $m$ control functions $\boldsymbol{u}(t)$ that minimize the performance index

$$
J=\varphi\left(\boldsymbol{x}_{f}, t_{f}\right)+\int_{t_{i}}^{t_{f}} L(\boldsymbol{x}(t), \boldsymbol{u}(t), t) d t
$$


The initial state vector, $\boldsymbol{x}_{i}$, and the final state vector, $\boldsymbol{x}_{f}$, are not necessarily fixed, as well as the final time $t_{f}$. In addition to the previous statements, boundary and path constraints can be imposed:

$$
\boldsymbol{\psi}\left(\boldsymbol{x}_{f}, t_{f}\right)=0 \text { and } \boldsymbol{C}(\boldsymbol{u}(t), t) \leq 0
$$

respectively, where $\boldsymbol{\psi}=\left\{\psi_{1}, \ldots, \psi_{p}\right\}$ and $\boldsymbol{C}=\left\{C_{1}, \ldots, C_{q}\right\}$.

The above problem can be solved by reformulating it as a boundary value problem on a set of differential algebraic equations (DAEs) [2]. To this aim, the dynamics and constraints are added to the performance index $J$ to form the so-called augmented performance index

$$
\begin{aligned}
\bar{J}= & \varphi\left(\boldsymbol{x}_{f}, t_{f}\right)+\boldsymbol{\nu}^{T} \boldsymbol{\psi}\left(\boldsymbol{x}_{f}, t_{f}\right)+ \\
& +\int_{t_{i}}^{t_{f}}\left[L(\boldsymbol{x}, \boldsymbol{u}, t)+\boldsymbol{\lambda}^{T}(\boldsymbol{f}(\boldsymbol{x}, \boldsymbol{u}, t)-\dot{\boldsymbol{x}})+\boldsymbol{\mu}^{T} \boldsymbol{C}(\boldsymbol{u}, t)\right] d t
\end{aligned}
$$

where two kind of Lagrange multipliers are introduced:

- a $p$-dimensional vector of constants, $\boldsymbol{\nu}$, for the final constraints in (7);

- an $n$-dimensional and a $q$-dimensional vector of functions $\boldsymbol{\lambda}$ and $\boldsymbol{\mu}$ for the dynamics in (5) and the path constraints in (7), which are usually referred to as adjoint or costate variables.

The optimal control problem is then reduced to identifying a stationary point of the augmented performance index $\bar{J}$. This is achieved by imposing the gradient of $\bar{J}$ to be zero with respect to all optimization variables; specifically, the state vector $\boldsymbol{x}$ and the control vector $\boldsymbol{u}$, the Lagrange multipliers $\boldsymbol{\nu}$ and the costate variables $\boldsymbol{\lambda}$ and $\boldsymbol{\mu}$, the unknown components of the initial state $\boldsymbol{x}_{i}$ and the final state $\boldsymbol{x}_{f}$, and the final time $t_{f}$. In particular, the optimality 
with respect to $\boldsymbol{\lambda}$ and $\boldsymbol{x}$ leads to the following relations:

$$
\begin{aligned}
& \frac{\partial \bar{J}}{\partial \boldsymbol{\lambda}}=0 \Rightarrow \dot{\boldsymbol{x}}=\boldsymbol{f}(\boldsymbol{x}, \boldsymbol{u}, t) \\
& \frac{\partial \bar{J}}{\partial \boldsymbol{x}}=0 \Rightarrow \dot{\boldsymbol{\lambda}}=-\left(\frac{\partial \boldsymbol{f}}{\partial \boldsymbol{x}}\right)^{T} \boldsymbol{\lambda}-\left(\frac{\partial L}{\partial \boldsymbol{x}}\right)^{T},
\end{aligned}
$$

whereas $\partial \bar{J} / \partial \boldsymbol{u}=0$ yields

$$
\left(\frac{\partial L}{\partial \boldsymbol{u}}\right)^{T}+\left(\frac{\partial \boldsymbol{f}}{\partial \boldsymbol{u}}\right)^{T} \boldsymbol{\lambda}+\left(\frac{\partial \boldsymbol{C}}{\partial \boldsymbol{u}}\right)^{T} \boldsymbol{\mu}=\mathbf{0} .
$$

Equations (9) and (10) together are usually referred to as Euler-Lagrange equations. It is worth observing that the Euler-Lagrange equations form a system of DAEs: the differential part is represented by Eq. (9), which defines the dynamics for the state variables $\boldsymbol{x}$ and the costate variables $\boldsymbol{\lambda}$; the role of the algebraic constraint is played by Eq. (10). The previous system must be coupled with the boundary conditions ensuing from the optimality conditions with respect to the remaining optimization variables (see [2] for further details). The optimal control problem is therefore solved as a boundary value problem on a system of DAEs.

A particular optimal control problem is addressed in this work. The dynamics is supposed to be affine in the control vector $\boldsymbol{u}$; i.e.

$$
\dot{\boldsymbol{x}}=\boldsymbol{f}(\boldsymbol{x}, \boldsymbol{u}, t)=\tilde{\boldsymbol{f}}(\boldsymbol{x}, t)+\mathrm{B}(\boldsymbol{x}) \boldsymbol{u},
$$

where $\mathrm{B}(\boldsymbol{x})$ is a $v \times m$ matrix, whose elements do not depend on the controls. Moreover the control functions are sought to minimize the performance index

$$
J=\frac{1}{2} \int_{t_{i}}^{t_{f}} \boldsymbol{u}^{T} \boldsymbol{u} d t
$$


and no path constraints are imposed. Based on the previous hypotheses, Eq. (10) assumes the simpler form

$$
\boldsymbol{u}+\mathrm{B}^{T}(\boldsymbol{x}) \boldsymbol{\lambda}=0
$$

Equation (13) supplies an explicit relation between the control functions $\boldsymbol{u}$ and the costate variables $\boldsymbol{\lambda}$, which can be substituted in Eq. (9). The original system of DAEs of the Euler-Lagrange equations translates into the system of ODEs

$$
\begin{aligned}
\dot{\boldsymbol{x}} & =\tilde{\boldsymbol{f}}(\boldsymbol{x}, t)-\mathrm{B}(\boldsymbol{x}) \mathrm{B}^{T}(\boldsymbol{x}) \boldsymbol{\lambda} \\
\dot{\boldsymbol{\lambda}} & =-\left(\frac{\partial \boldsymbol{f}(\boldsymbol{x}, \boldsymbol{\lambda}, t)}{\partial \boldsymbol{x}}\right)^{T} \boldsymbol{\lambda} .
\end{aligned}
$$

Therefore, the original optimal control problem reduces to a two-point boundary value problem on the set of ODEs (14), where boundary conditions are imposed on the initial and final values of the state and costate variables, depending on the optimal control problem at hand.

\section{High Order Optimal Feedback}

Suppose the problem of transferring a spacecraft from a fixed initial state to a fixed final state with fixed $t_{i}$ and $t_{f}$ is of interest; i.e., boundary conditions assume the simpler form

$$
\left\{\begin{array}{l}
\boldsymbol{x}_{i}=\overline{\boldsymbol{x}}_{i} \\
\boldsymbol{x}_{f}=\overline{\boldsymbol{x}}_{f} .
\end{array}\right.
$$

The optimal control problem is then reduced to the problem of solving Eq. (14) subject to the boundary conditions in Eq. (15).

Several techniques are available in the literature to solve the previous problem for assigned $\overline{\boldsymbol{x}}_{i}$ and $\overline{\boldsymbol{x}}_{f}$, like the simple and multiple shooting schemes 
or difference methods [15]. This means that, given $\overline{\boldsymbol{x}}_{i}$ and $\overline{\boldsymbol{x}}_{f}$, the previous techniques are applied to compute the initial values of the costate variables that solve the TPBVP, which will be indicated as $\boldsymbol{\lambda}_{i}^{0}$. The solution is then uniquely identified by the initial state and costate vectors, $\overline{\boldsymbol{x}}_{i}$ and $\boldsymbol{\lambda}_{i}^{0}$ respectively.

Assume now a reference solution $\boldsymbol{\lambda}_{i}^{0}$ is available and suppose the Taylor expansion of the solution of the optimal control problem with respect to the initial state and the final state is of interest. Differential algebra can effectively serve this purpose. To this aim, initialize both the initial state $\boldsymbol{x}_{i}$ and the initial costate $\boldsymbol{\lambda}_{i}$ as DA variables. This means the variations

$$
\begin{aligned}
& {\left[\boldsymbol{x}_{i}\right]=\overline{\boldsymbol{x}}_{i}+\delta \boldsymbol{x}_{i}} \\
& {\left[\boldsymbol{\lambda}_{i}\right]=\boldsymbol{\lambda}_{i}^{0}+\delta \boldsymbol{\lambda}_{i}}
\end{aligned}
$$

with respect to the fixed initial state $\overline{\boldsymbol{x}}_{i}$ and the reference solution $\boldsymbol{\lambda}_{i}^{0}$ are considered.

Using the techniques introduced in Sect. 3, the solution of Eq. (14) at $t_{f}$ is expanded with respect to the initial state and costate vectors. More specifically, the dependence of the final state and costate vectors on their initial values are obtained in terms of the high order polynomial map

$$
\left(\begin{array}{c}
{\left[\boldsymbol{x}_{f}\right]} \\
{\left[\boldsymbol{\lambda}_{f}\right]}
\end{array}\right)=\left(\begin{array}{c}
\overline{\boldsymbol{x}}_{f}+\delta \boldsymbol{x}_{f} \\
\boldsymbol{\lambda}_{f}^{0}+\delta \boldsymbol{\lambda}_{f}
\end{array}\right)=\left(\begin{array}{c}
\overline{\boldsymbol{x}}_{f} \\
\boldsymbol{\lambda}_{f}^{0}
\end{array}\right)+\left(\begin{array}{c}
\mathcal{M}_{\boldsymbol{x}_{f}} \\
\mathcal{M}_{\boldsymbol{\lambda}_{f}}
\end{array}\right)\left(\begin{array}{c}
\delta \boldsymbol{x}_{i} \\
\delta \boldsymbol{\lambda}_{i}
\end{array}\right),
$$

where $\overline{\boldsymbol{x}}_{f}$ and $\boldsymbol{\lambda}_{f}^{0}$ are the constant part of the map (i.e., the reference solution flowing from $\overline{\boldsymbol{x}}_{i}$ and $\boldsymbol{\lambda}_{i}^{0}$ under the ODEs (14)), whereas all higher order terms are included in $\mathcal{M}_{\boldsymbol{x}_{f}}$ and $\mathcal{M}_{\boldsymbol{\lambda}_{f}}$. 
Subtract now the constant part from Eq. (17) for

$$
\left(\begin{array}{c}
\delta \boldsymbol{x}_{f} \\
\delta \boldsymbol{\lambda}_{f}
\end{array}\right)=\left(\begin{array}{c}
\mathcal{M}_{\boldsymbol{x}_{f}} \\
\mathcal{M}_{\boldsymbol{\lambda}_{f}}
\end{array}\right)\left(\begin{array}{c}
\delta \boldsymbol{x}_{i} \\
\delta \boldsymbol{\lambda}_{i}
\end{array}\right) .
$$

Then, extract $\mathcal{M}_{\boldsymbol{x}_{f}}$ from Eq. (18) and consider the map

$$
\left(\begin{array}{c}
\delta \boldsymbol{x}_{f} \\
\delta \boldsymbol{x}_{i}
\end{array}\right)=\left(\begin{array}{c}
\mathcal{M}_{\boldsymbol{x}_{f}} \\
\mathcal{I}_{\boldsymbol{x}_{i}}
\end{array}\right)\left(\begin{array}{c}
\delta \boldsymbol{x}_{i} \\
\delta \boldsymbol{\lambda}_{i}
\end{array}\right),
$$

which is built by concatenating $\mathcal{M}_{\boldsymbol{x}_{f}}$ with the identity map for $\delta \boldsymbol{x}_{i}, \mathcal{I}_{\boldsymbol{x}_{i}}$.

Using inversion techniques for high order polynomials [12], the map in Eq. (19) is inverted to obtain

$$
\left(\begin{array}{c}
\delta \boldsymbol{x}_{i} \\
\delta \boldsymbol{\lambda}_{i}
\end{array}\right)=\left(\begin{array}{c}
\mathcal{M}_{\boldsymbol{x}_{f}} \\
\mathcal{I}_{\boldsymbol{x}_{i}}
\end{array}\right)^{-1}\left(\begin{array}{c}
\delta \boldsymbol{x}_{f} \\
\delta \boldsymbol{x}_{i}
\end{array}\right) .
$$

Consider now the components of map (20) for $\delta \boldsymbol{\lambda}_{i}$, which will be indicated as

$$
\delta \boldsymbol{\lambda}_{i}=\mathcal{M}_{\boldsymbol{\lambda}_{i}}\left(\delta \boldsymbol{x}_{f}, \delta \boldsymbol{x}_{i}\right) .
$$

The polynomials in (21) are the arbitrary order Taylor expansion of the solution of the optimal control problem with respect to the initial and final states. More specifically, given any perturbation $\delta \boldsymbol{x}_{i}$ and $\delta \boldsymbol{x}_{f}$ of the initial and final state from their reference values $\overline{\boldsymbol{x}}_{i}$ and $\overline{\boldsymbol{x}}_{f}$, the mere evaluation of the polynomials in Eq. (21) delivers the high order correction $\delta \boldsymbol{\lambda}_{i}$ to $\boldsymbol{\lambda}_{i}^{0}$ to obtain the corresponding solution of the optimal control problem from the perturbed initial state $\overline{\boldsymbol{x}}_{i}+\delta \boldsymbol{x}_{i}$ to the perturbed final state $\overline{\boldsymbol{x}}_{f}+\delta \boldsymbol{x}_{f}$.

It is worth observing that a possible alternative approach to address the previous problem consists in solving the TPBVP for the new solution $\boldsymbol{\lambda}_{i}$ using classical techniques. The main disadvantage of this approach is that a 
new TPBVP must be solved for each displaced initial and final states. This involves running through the iterative procedures of the classical TPBVP solvers. Each iterative procedure is able to deliver one solution, whose validity is limited to one specific $\delta \boldsymbol{x}_{i}$ and $\delta \boldsymbol{x}_{f}$. Consequently, the classical TPBVP solvers should be applied for each new $\delta \boldsymbol{x}_{i}$ and $\delta \boldsymbol{x}_{f}$. The Taylor expansion of the optimal control problem supplies an effective alternative method to overcome this issue. First of all, analytical information is gained, which can supply a valuable insight on the underlying dynamics. Moreover, for any $\delta \boldsymbol{x}_{i}$ and $\delta \boldsymbol{x}_{f}$, the same polynomial map is evaluated to compute the optimal control law connecting the displaced initial state to the displaced target state. This means that the high order map in Eq. (21) must be computed only once for all possible offsets, and the optimal control laws are then obtained through the evaluation of the same polynomials, so avoiding the use of iterative algorithms. Nevertheless, the polynomial relation between $\delta \boldsymbol{\lambda}_{i}$, and $\delta \boldsymbol{x}_{i}$ and $\delta \boldsymbol{x}_{f}$ given by Eq. (21) is accurate up to the order of the DA-based computation.

\section{Applications}

The performances of the high order optimal feedback control method introduced in Sect. 5 are investigated on three test cases: a rendezvous maneuver, the landing of a probe on Moon's surface, and a continuously propelled Earth-Mars transfer. The effectiveness of the control corrections, as well as the accuracy of the polynomial expansions, and the computational efficiency of the method are assessed. The three test cases favor the illustration of the performances of the method and have been taken from relevant 
literature [17]. Large perturbations on boundary conditions are considered to magnify nonlinearities and properly compare the high order solutions with those attained using classical nonlinear methods such as simple shooting.

As mentioned above, the accuracy of map (21) depends on the computation order. Within the radius of convergence of the Taylor expansion, the order necessary to meet a given accuracy level can be selected by assessing the error of the polynomial approximation. The error of a Taylor expansion of order $n$ over the set of all admissible perturbations can be estimated by computing the range of the polynomial of all $n+1$-st order terms over the same set. This can be done using polynomial bounders available in COSYInfinity [14]. The same technique can be adopted to estimate the level of uncertainty that can be managed for a given expansion order and desired accuracy. This procedure was adopted to select the expansion order used in the lunar landing test case. On the other hand, the dependency of the error on the expansion order is studied in the rendezvous and Earth-Mars transfer test cases.

\subsection{Rendezvous Maneuver}

A rendezvous maneuver is analyzed as the first test case for the high order optimal feedback technique introduced in Sect. 5. The study of this problem is motivated by the work of Park, Guibout and Scheeres based on the alternative approach of generating functions $[17,11]$. The space rendezvous is a maneuver which takes two spacecraft, originally moving on different orbits, to the same final reference orbit, matching their positions and velocities. Referring to Fig. 2, this rather general case can be focused on the problem of a spacecraft (referred to as chaser) targeting an object (referred to as target) 


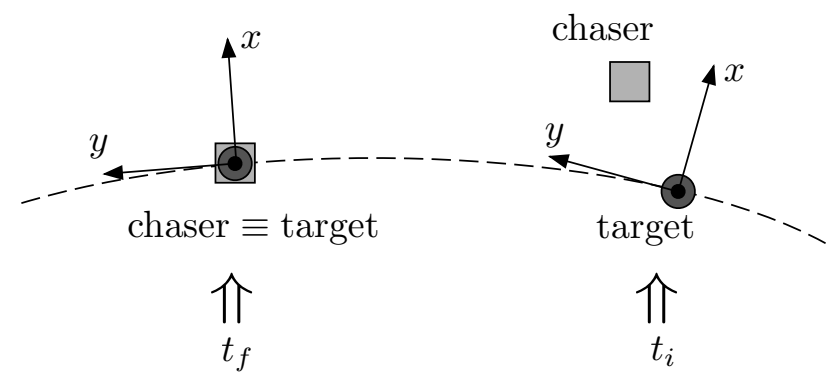

Figure 2: Rendezvous maneuver.

on its orbit.

A continuously propelled rendezvous maneuver is considered. The target is supposed to move on a circular orbit of radius $R$, whereas the chaser is assumed to be subject to a controlled two-body dynamics. In this framework, the rendezvous maneuver is classically designed in a non-inertial reference frame that is centered at the target position, with $x$-axis constantly aligned with the orbital radius, $y$-axis directed towards the target orbital velocity, and $z$-axis chosen to form a right-handed coordinate system with $x$ and $y$ (see Fig. 2). Thus, the non-inertial reference frame rotates along the circular target orbit with constant angular velocity $\omega$ and the chaser is subject to the relative dynamics

$$
\begin{aligned}
& \dot{x}=v_{x}, \quad \dot{y}=v_{y}, \quad \dot{z}=v_{z} \\
& \dot{v}_{x}=2 \dot{y}-(1+x)\left(\frac{1}{r^{3}}-1\right)+u_{x} \\
& \dot{v}_{y}=-2 \dot{x}-y\left(\frac{1}{r^{3}}-1\right)+u_{y} \\
& \dot{v}_{z}=-\frac{1}{r^{3}} z+u_{z},
\end{aligned}
$$

where lengths and time are normalized using $R$ and $1 / \omega$, respectively; $\boldsymbol{u}=$ $\left(u_{x}, u_{y}, u_{z}\right)$ is the control vector; and $r=\sqrt{(1+x)^{2}+y^{2}+z^{2}}$. 
The chaser is supposed to have initial offsets from the target in both position and velocity, which are denoted by $\delta \boldsymbol{r}_{i}$ and $\delta \boldsymbol{v}_{i}$ respectively. The optimal control problem is solved to design the control law $\boldsymbol{u}$ that takes the chaser from its displaced initial state to the fixed target state (i.e., to the origin of the rotating frame with zero velocity) in a given time $t_{f}-t_{i}$. The relative dynamics $(22)$ is affine in the control vector $\boldsymbol{u}$. Thus, the optimal control problem can be reduced to a TPBVP with fixed initial and final states for the chaser.

First, a reference solution of the optimal control problem must be identified before applying the high order DA-based technique. To this aim, it is worth observing that the relative dynamics in Eq. (22) satisfies $\boldsymbol{f}(\boldsymbol{x}=0, \boldsymbol{u}=$ $0, t)=0$, with $\boldsymbol{x}=\left(x, y, z, v_{x}, v_{y}, v_{z}\right)$. This means that $\boldsymbol{x}(t)=0$ and $\boldsymbol{u}(t)=0$ for any $t$ is a trivial solution of the optimal control problem that is used as reference solution for the high order expansion.

The performances of the high order optimal feedback control algorithm are now investigated. The chaser is supposed to have a displaced initial position $\delta \boldsymbol{r}_{i}=(0.2,0.2,0)$ and a displaced initial velocity $\delta \boldsymbol{v}_{i}=(0.1,0.1,0)$. The rendezvous maneuver is designed to take the chaser to the target state in 1 time unit. The exact solution of the optimal control problem is first identified by solving the associated TPBVP using a simple shooting technique. The result is reported in Figs. 3-5 in terms of position, velocity, and control profile, respectively. It is worth observing that the relative dynamics in Eq. (22), together with Eq. (13), yields $\boldsymbol{u}=-\boldsymbol{\lambda}_{4,5,6}$, so that $\lambda_{4}=-u_{x}$, $\lambda_{5}=-u_{y}$, and $\lambda_{6}=-u_{z}$. For the sake of completeness, Fig. 6 illustrates the reference profile of the first two components of the costate vector, $\boldsymbol{\lambda}_{1,2}$. 

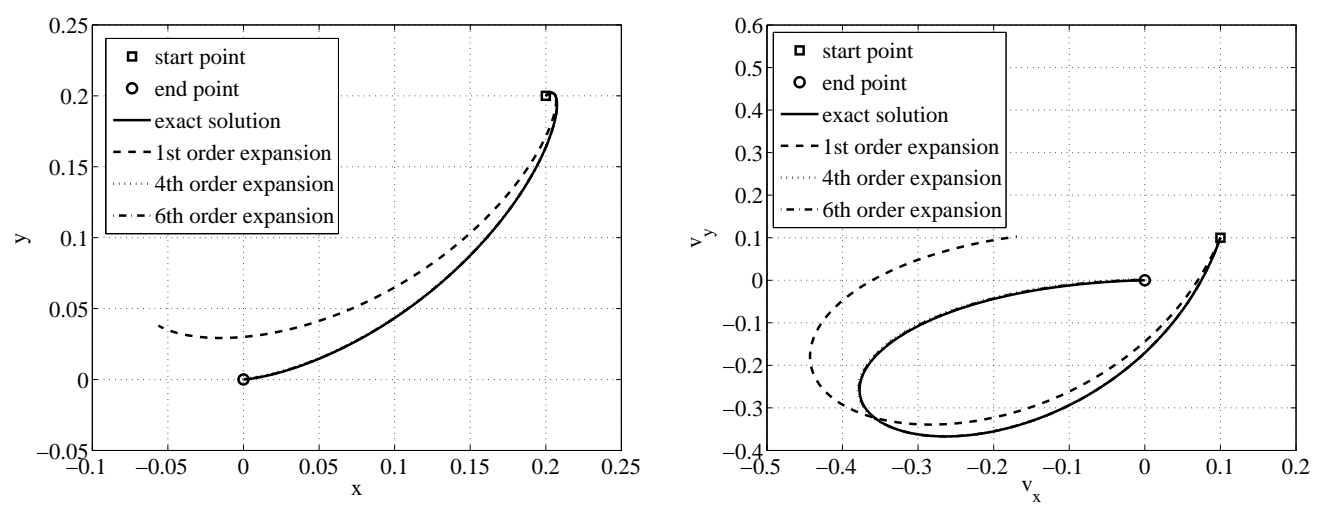

Figure 3: Rendezvous maneuver: position. Figure 4: Rendezvous maneuver: velocity.

Figures 3 and 4 show that the exact solution takes the chaser to the target state in the assigned time. The exact solution is then compared with the trajectory, control, and costate profiles obtained using the DA-based optimal feedback control algorithm introduced in Sect. 5, using different expansion orders. As can be seen, the low accuracy of the 1-st order correction is significantly improved using 4-th and 6-th order expansions. This is confirmed in Figs. 7 and 8, which illustrate the profiles of the error of the DA-based approximation with respect to the exact solution along the maneuver at different expansion orders. The error is computed as the norm of the difference vector between the DA-based approximation of $\boldsymbol{u}$ and $\boldsymbol{\lambda}_{1,2,3}$, and their exact counterparts.

As already mentioned in Sect. 5, the main advantage of the high order optimal feedback control algorithm is that map (21) must be computed only once for all possible offsets: for any initial offset of the chaser with respect to the target, the same polynomial map is evaluated to compute the corresponding optimal control law. Then, moving to the real scenario, once the 


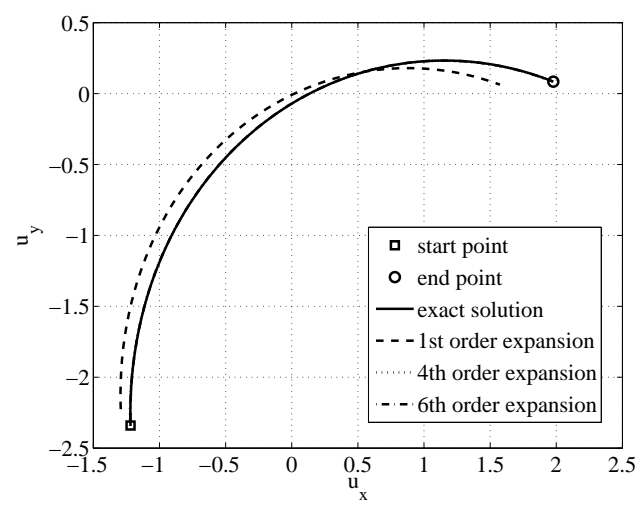

Figure 5: Rendezvous: control.

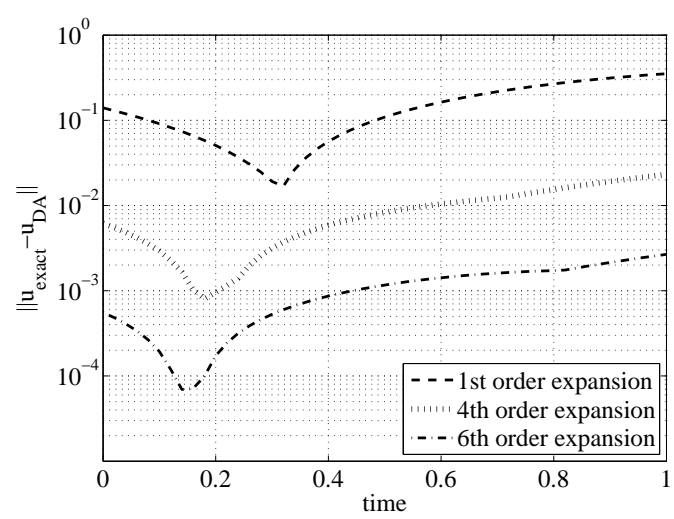

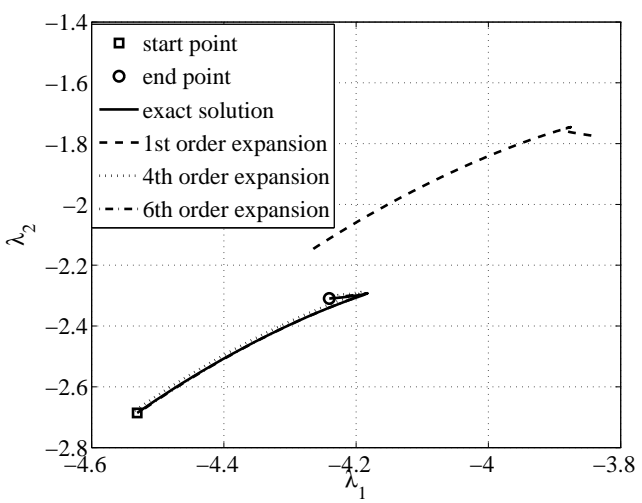

Figure 6: Rendezvous: $\boldsymbol{\lambda}_{1,2}$.

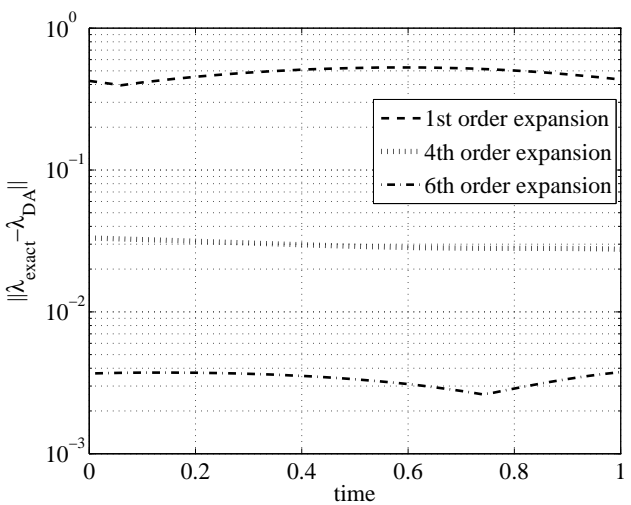

Figure 7: Rendezvous: control error with Figure 8: Rendezvous: $\boldsymbol{\lambda}_{1,2,3}$ error with rerespect to the exact solution. spect to the exact solution.

offset is measured, the corresponding optimal control can be computed by evaluating map (21) instead of using iterative techniques. This feature is exploited in Fig. 9 and Fig. 10: a set of 20 perturbed positions distributed over a circle of radius 0.2 in the rotating frame is selected. For each sample, a 6 -th order correction is computed using the polynomial map (21). Clearly, the chaser is always moved to the origin of the reference frame. 
The advantages of the high order optimal feedback with respect to classical simple shooting is now assessed. The computational time required to evaluate the 6 -th order map (21) at all perturbed positions is $7.18 \cdot 10^{-3} \mathrm{~s}$ on a Intel Core i5 2.4 GHz, running Mac OS X 10.7.5. This value can be compared with the time required to obtain the exact solution for all perturbed positions using classical simple shooting. Using ballistic motion to compute the first guess for the initial costate vector, the iterative procedure to solve the optimal control problem for all perturbed initial positions using simple shooting takes $13.62 \mathrm{~s}$ on the same machine. Although more efficient procedures might be used to identify first guesses for the initial costates, the significant difference in computational time highlights the advantage of high order optimal feedback control with respect to classical simple shooting. The time required to compute the 6-th order map (21) is 5.75 s. However, as mentioned above, it must be computed only once for all possible offsets, which is performed offline.

The radius of convergence of the Taylor expansion is investigated in Figs. 11 and 12. More specifically, a radial displacement of the chaser initial position of the form $\delta \boldsymbol{r}_{i}=(r, 0,0)$ is imposed, whereas the initial velocity is set to its reference value (i.e., $\left.\delta \boldsymbol{v}_{i}=(0,0,0)\right)$. The error of the DA-based approximation with respect to the exact solution is computed as the norm of the difference vectors between the associated initial values of $\boldsymbol{u}$ and $\boldsymbol{\lambda}_{1,2,3}$. The errors on $\boldsymbol{u}$ and $\boldsymbol{\lambda}_{1,2,3}$ are reported in Figs. 11 and 12 respectively, for different expansion orders. As expected, the error of the DA-based approximation tends to increase with the distance $r$ from the reference position. The convergence radius can be estimated as the maximum $r$ for which the error 

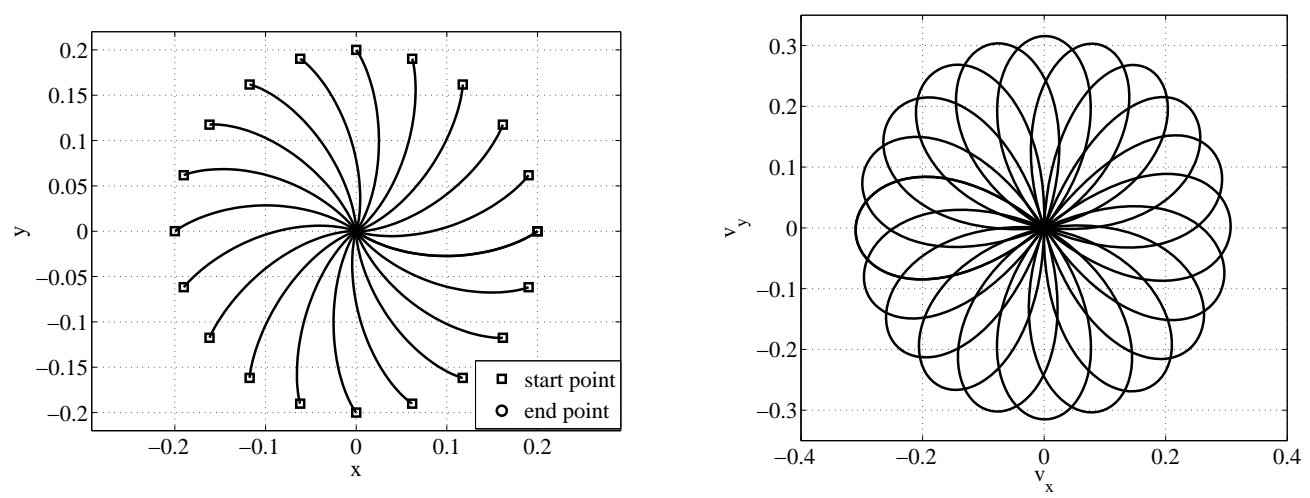

Figure 9: Rendezvous: positional trajecto- Figure 10: Rendezvous: velocity trajectories ries for initial positions lying on a circle of for initial positions lying on a circle of radius radius 0.2 .

0.2. Each petal is flown clockwise.
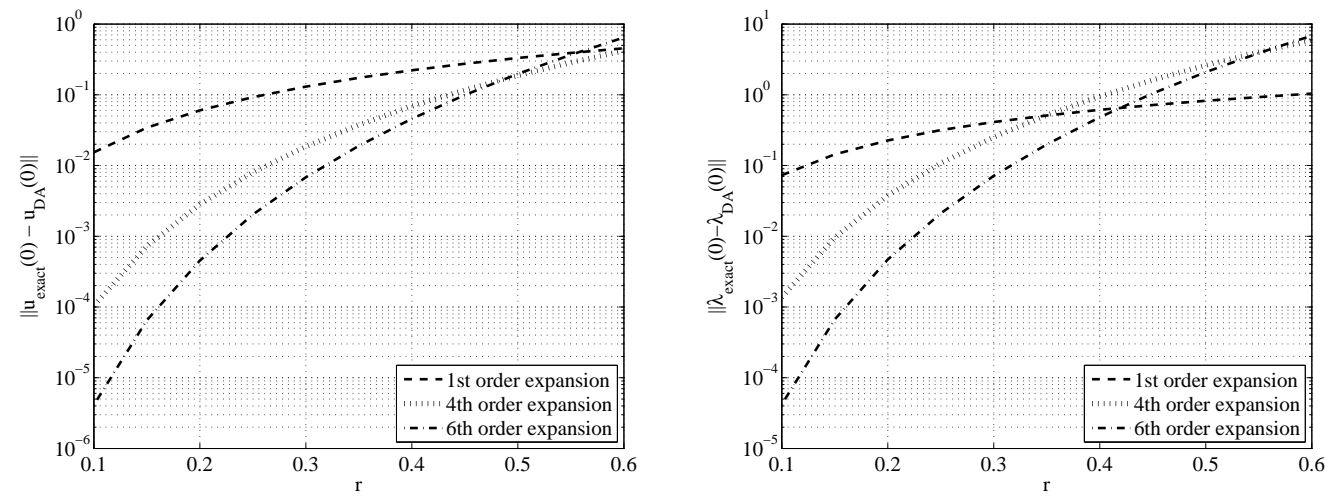

Figure 11: Rendezvous: error of the DA- Figure 12: Rendezvous: error of the DAbased approximation on the initial control based approximation on the initial $\boldsymbol{\lambda}_{1,2,3} \mathrm{vs}$. vs. distance from reference point. distance from reference point.

tends to decrease for increasing expansion orders. It can be approximated to about 0.35 in this test case. 


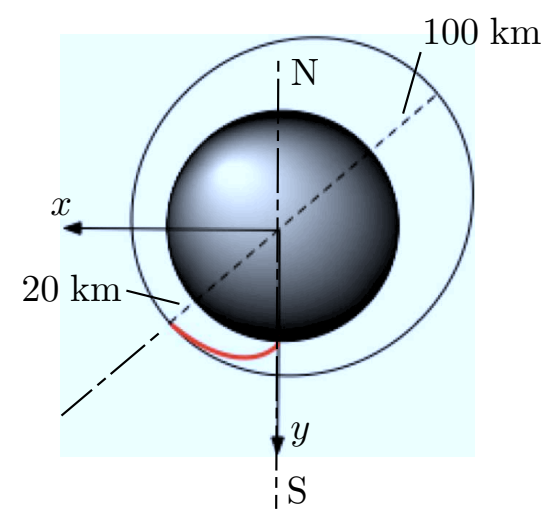

Figure 13: Lunar landing problem.

\subsection{Lunar Landing}

The optimal feedback control of a probe landing on Moon's South pole is addressed in this section. The control profile is designed in the frame of the controlled two-body problem. Referring to Fig. 13, the lander is supposed to originally move on an elliptical polar descent orbit, taking it from an altitude of $100 \mathrm{~km}$ (apocenter) to an altitude of $20 \mathrm{~km}$ (pericenter). The landing phase is supposed to start at the pericenter of the descent orbit. Final conditions are imposed to put the lander over Moon's south pole at an altitude of $2 \mathrm{~m}$, with a downward velocity of $3 \mathrm{~m} / \mathrm{s}$, from which the final phase of the landing maneuver is supposed to start. A Cartesian reference frame is selected to describe the dynamics: the $y$-axis is aligned with Moon's South pole; the $x$ axis lies on Moon's equatorial plane, pointing towards the orbital descending node; the $z$-axis is selected to form a right-handed reference system. The landing dynamics is described by the set of ODEs:

$$
\begin{aligned}
\dot{\boldsymbol{r}} & =\boldsymbol{v} \\
\dot{\boldsymbol{v}} & =-\frac{\mu}{r^{3}} \boldsymbol{r}+\boldsymbol{u}
\end{aligned}
$$


in which $\boldsymbol{r}$ and $\boldsymbol{v}$ are the probe position and velocity, respectively; $r=\|\boldsymbol{r}\|$; $\mu$ is Moon's gravitational parameter; and $\boldsymbol{u}$ is the control vector. As from Eq. (23), the dynamics is affine in the control vector $\boldsymbol{u}$. Thus, Eq. (14) holds for the problem at hand and the optimal control problem is then reduced to a TPBVP with fixed initial and final states for the landing probe.

A reference solution of the optimal control problem is first identified by solving the resulting TPBVP. The initial time is chosen to be zero, whereas the landing duration is set to $31 \mathrm{~min}$. A simple shooting technique is used to solve the TPBVP and to compute the reference trajectory reported in Fig. 14. Figure 15 illustrates the corresponding reference control profile in terms of histories of its components. Due to the symmetry of the problem, the reference trajectory lies on the $x-y$ plane. Similarly to the rendezvous test case, within the dynamics of Eq. (23), Eq. (13) yields $\boldsymbol{u}=-\boldsymbol{\lambda}_{4,5,6}$, where $\boldsymbol{\lambda}_{4,5,6}$ are the last three components of the costate vector. Thus, Fig. 15 is also representative of the reference profile for $\boldsymbol{\lambda}_{4,5,6}$. For the sake of completeness, Fig. 16 illustrates the reference profile of the remaining three components of the costate vector, $\boldsymbol{\lambda}_{1,2,3}$, which can be shown to equal the first derivative of the control profile [16].

The initial probe position and velocity, $\boldsymbol{r}_{i}$ and $\boldsymbol{v}_{i}$ respectively, are now supposed to be affected by errors. The high order optimal feedback control algorithm introduced in Sect. 5 is applied to optimally correct the control law in order to reach the reference final state. More specifically, the reference trajectory in Fig. 14 is used as reference solution for the Taylor expansions. The algorithm is then applied to compute the polynomial map (21) for the problem at hand using third order expansions. Thus, given any perturbation 


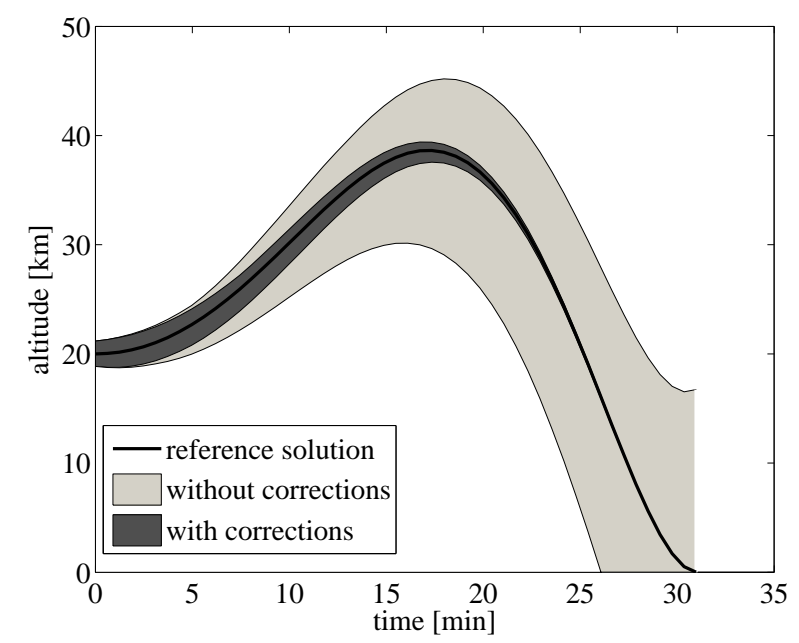

Figure 14: Lunar landing: altitude dispersion.
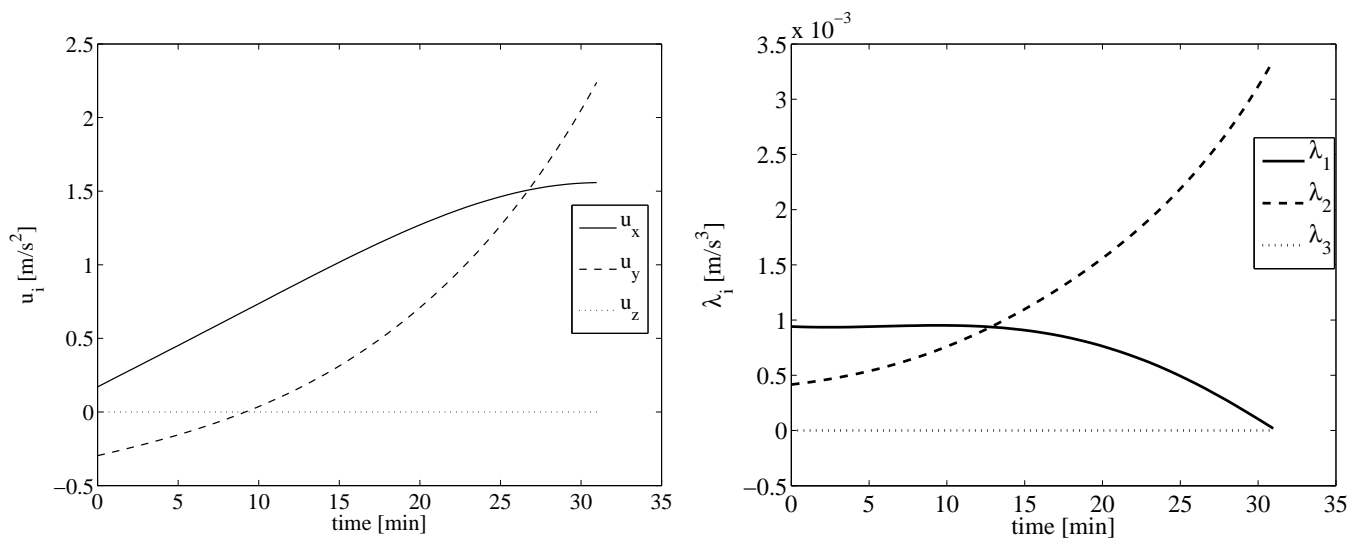

Figure 15: Lunar landing: reference control Figure 16: Lunar landing: reference $\boldsymbol{\lambda}_{1,2,3}$ profile. profile.

$\delta \boldsymbol{r}_{i}$ and $\delta \boldsymbol{v}_{i}$, the polynomial map is evaluated by setting $\delta \boldsymbol{x}_{i}=\left(\delta \boldsymbol{r}_{i}, \delta \boldsymbol{v}_{i}\right)$ and $\delta \boldsymbol{x}_{f}=(0,0)$. The corresponding optimal value of $\boldsymbol{\lambda}_{i}$ is computed.

The performances of the procedure are studied hereafter. A maximum position error of $1 \mathrm{~km}$ and a maximum velocity error of $5 \mathrm{~m} / \mathrm{s}$ are supposed to 
affect each component of the initial lander position and velocity, respectively. The final dispersion at landing is then investigated. First of all, for the sake of a more complete analysis, given any perturbed initial conditions, no corrections to the nominal costate variables (and, consequently, to the control) are applied. In particular, 100 samples are randomly generated within the initial uncertainty box with uniform distribution. Each sample is then propagated using the nominal guidance law. The resulting maximum and minimum lander altitudes at each integration time are computed over the propagated set. Figure 14 shows the resulting altitude dispersion throughout landing. The figure illustrates how initial conditions corresponding to both impacts on Moon's surface (lower area of the strip) and trajectories moving away from the landing site (higher area of the strip) are included in the initial error box.

The high order optimal feedback is then applied. The third order corrections are computed using Eq. (21): for the same random samples of Fig. 14, the errors on the initial state are computed and the map is evaluated to correct the reference $\boldsymbol{\lambda}_{i}^{0}$. The resulting set of trajectories is reported again in Fig. 14 for the sake of comparison. The corrected optimal control laws take the probe to the final desired conditions and the resulting final dispersion is drastically reduced. This is better illustrated in Figs. 17 and 18, where the fulfillment of the requirements on the final state vector is investigated. For each sample, the error of the corresponding trajectory with respect to the reference one is evaluated in terms of displacements of the position and velocity vectors from their reference values. More precisely, at each integration time, the position and velocity errors are computed as maximum norms of 


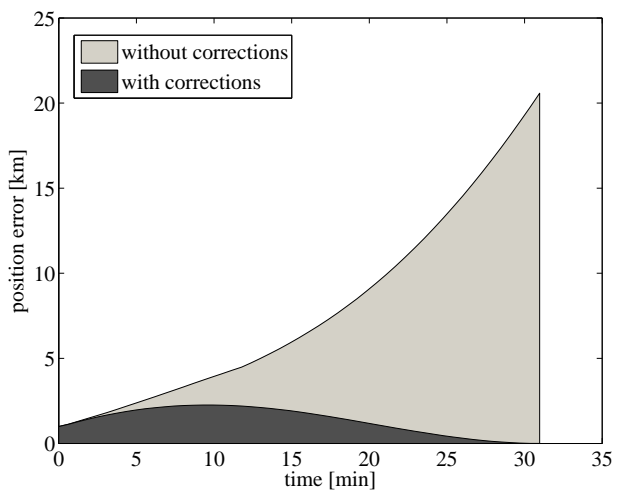

Figure 17: Lunar landing: position error.

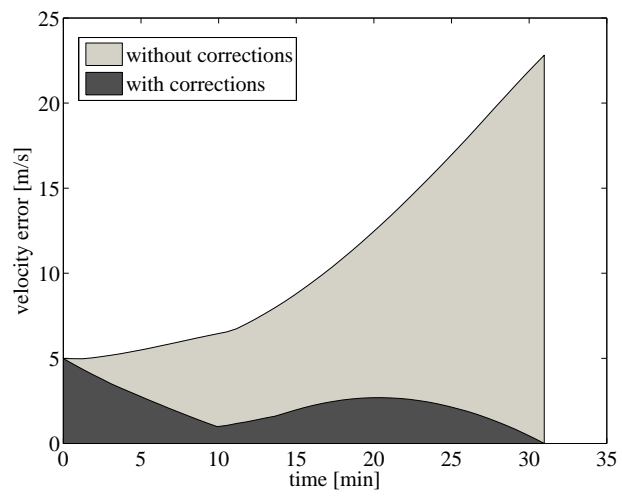

Figure 18: Lunar landing: velocity error.

the associated difference vectors. Thus, the maximum position and velocity errors are evaluated at each integration time over the propagated set. The resulting curves are used to identify the areas reported in Figs. 17 and 18. The maximum errors for the uncorrected reference control law (light grey) are compared with those achievable using the corrected costate variables (dark grey). Using the control corrections, the final position error is reduced to a maximum value of about $0.5 \cdot 10^{-1} \mathrm{~m}$, to compare to a maximum value of about $20 \mathrm{~km}$ without corrections. Similar results hold for the velocity error, which is reduced to a maximum value of about $1 \cdot 10^{-4} \mathrm{~m} / \mathrm{s}$ instead of a maximum value of $23 \mathrm{~m} / \mathrm{s}$. It is worth observing that, despite the small final errors, the displacements along the maneuver turn out to be large. This is a fair result, as constraints are imposed only on the final conditions.

The control corrections are analyzed in Fig. 19. For each component of the control vector $\boldsymbol{u}$, the maximum control correction is evaluated among the random samples, and the resulting curves are reported in figure. A maximum control correction of about $0.016 \mathrm{~m} / \mathrm{s}^{2}$ is required for the given error box. The 

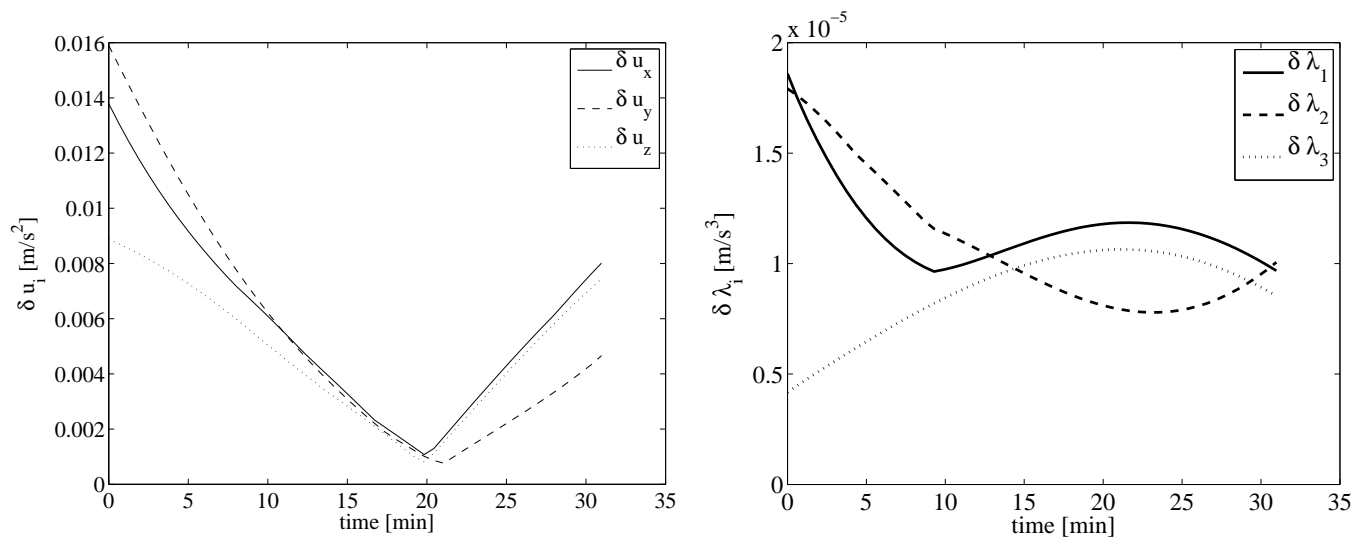

Figure 19: Lunar landing: maximum control Figure 20: Lunar landing: maximum $\boldsymbol{\lambda}_{1,2,3}$ corrections. corrections.

same approach is used to assess the maximum corrections to the reference values of $\boldsymbol{\lambda}_{1,2,3}$. The resulting profiles are reported in Fig. 20.

Despite the optimality of the feedback strategy, the value of the performance index in Eq. (12), as well as fuel consumption, varies depending on the control profiles. Table 1 compares the reference value of the performance index with its minimum and maximum values assumed over all the samples. The same comparison is carried out on the mass fraction

$$
\frac{m_{f}}{m_{i}}=\exp \left(-\frac{\int_{t_{i}}^{t_{f}}\|\boldsymbol{u}\| \mathrm{d} t}{I_{s p} g_{0}}\right),
$$

where $m_{i}$ and $m_{f}$ are the initial and final probe mass, respectively; $g_{0}$ is the standard gravity; $I_{s p}$ is the specific impulse of the thrusters, which is assumed to equal $317 \mathrm{~s}$.

\subsection{Earth-Mars Transfer}

The last test problem concerns a low-thrust Earth-Mars transfer. The transfer is designed in the frame of the controlled two-body problem. Con- 
Table 1: Lunar landing: performance index and mass fraction range over all samples.

\begin{tabular}{lccc}
\hline Parameter & Nom. value & Min. value & Max. value \\
\hline Performance index $\left[\mathrm{m}^{2} / \mathrm{s}^{3}\right]$ & 1124.300 & 1122.828 & 1126.148 \\
Mass fraction $m_{f} / m_{i}$ & 0.48126 & 0.48045 & 0.48210 \\
\hline
\end{tabular}

sequently, the spacecraft motion is modeled in the inertial ecliptic reference frame by the six first order ODEs

$$
\begin{aligned}
\dot{x} & =v_{x}, \quad \dot{y}=v_{y}, \quad \dot{z}=v_{z} \\
\dot{v}_{x} & =-\frac{\mu}{r^{3}} x+u_{x} \\
\dot{v}_{y} & =-\frac{\mu}{r^{3}} y+u_{y} \\
\dot{v}_{z} & =-\frac{\mu}{r^{3}} z+u_{z},
\end{aligned}
$$

where $r=\sqrt{x^{2}+y^{2}+z^{2}}, \mu$ is Sun's gravitational parameter, $\boldsymbol{u}=\left\{u_{x}, u_{x}, u_{z}\right\}$ is the control acceleration. Boundary constraints are imposed at the beginning and the end of the transfer. Specifically, the spacecraft is constrained to leave the Earth with Earth's velocity at time $t_{i}=1213.789$ MJD2000 and to match Mars' position and velocity at time $t_{f}$, where the transfer time $t_{f}-t_{i}$ is set to 513.210 days. Similarly to the previous test cases, the dynamics (25) is affine in the control. Thus, the optimal control problem can be reduced to a TPBVP with fixed initial and final states.

A reference optimal transfer is first identified by solving the TPBVP with a simple shooting technique. The resulting optimal transfer is illustrated in Fig. 21 in terms of a two-dimensional projection of the optimal trajectory on the ecliptic plane. The reference optimal control magnitude profile is reported in Fig. 22. 


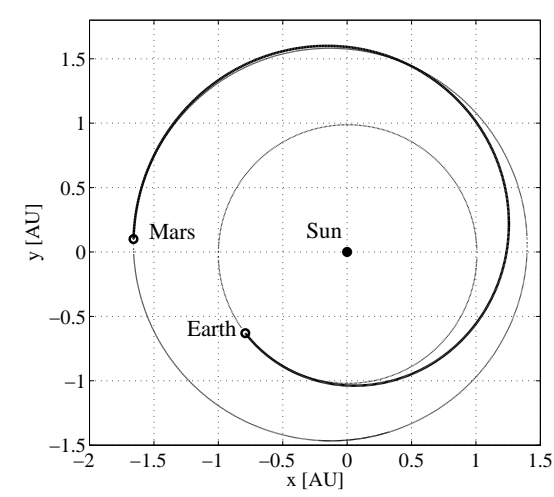

Figure 21: Reference optimal trajectory for Figure 22: Reference optimal control for the the Earth-Mars transfer problem.

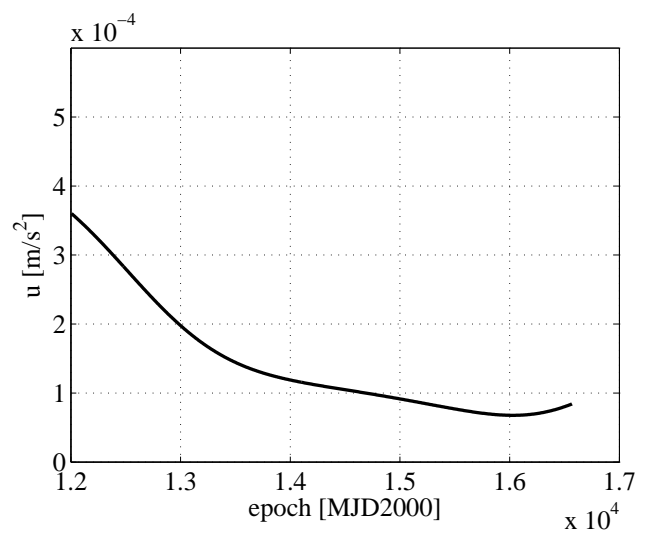

Earth-Mars transfer problem.

The problem of targeting a perturbed final state is now addressed. The third order Taylor expansion of the solution of the optimal transfer problem with respect to the final state is computed with the algorithm introduced in Sect. 5 to obtain a third order polynomial map (21). Then, the final position of the transfer is supposed to be affected by a maximum measurable error of $0.1 \mathrm{AU}$ on each component. Thus, given any perturbation $\delta \boldsymbol{r}_{f}$ of the final position from its reference value, the polynomial map (21) is evaluated at $\delta \boldsymbol{x}_{f}=\left(\delta \boldsymbol{r}_{f}, 0\right)$ and $\delta \boldsymbol{x}_{i}=(0,0)$. The corresponding optimal value of $\boldsymbol{\lambda}_{i}$ is computed. Then, starting from the reference initial spacecraft state and the new initial costates, a forward point-wise integration of the state and costate dynamics delivers the optimal control law and transfer trajectory from the reference initial state to the perturbed final target position.

The performances of the procedure are studied hereafter. A maximum perturbation of $0.1 \mathrm{AU}$ is imposed on the $x$ and $y$ components of the final 


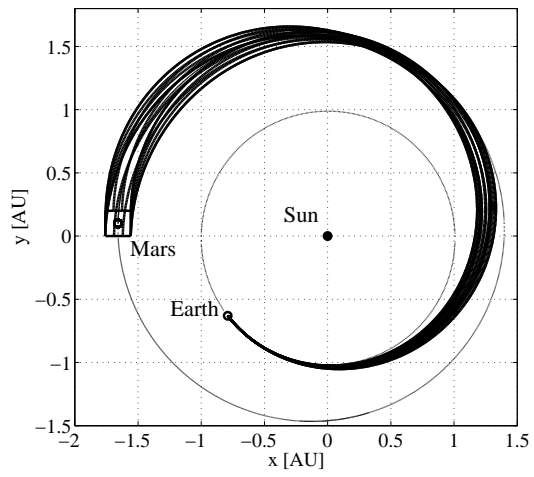

Figure 23: Earth-Mars transfer problem: Figure 24: Earth-Mars transfer problem: trajectories for third order corrections. detail of Fig. 23 at arrival.

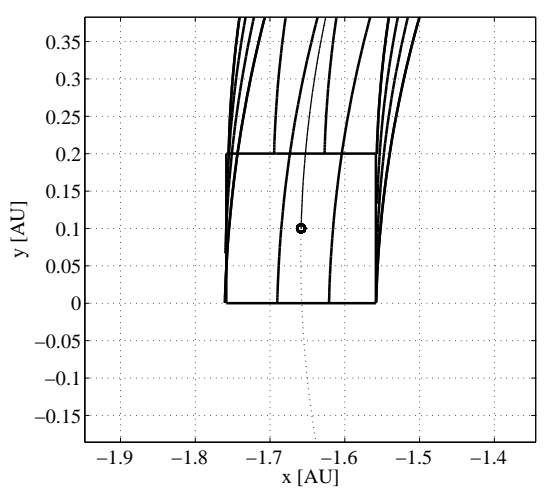

target position. The boundary of the corresponding square is sampled uniformly. For each sample, the associated $\delta \boldsymbol{r}_{f}$ is computed and the map (21) is evaluated to obtain the new optimal trajectory. The resulting transfers are reported in Fig. 23: starting from the reference initial position, the new trajectories move away from the reference one along the transfer and reach the imposed position on the final square (see Fig. 24 for a detail at arrival). Figure 25 plots the resulting optimal control magnitude profiles. Once again it is worth highlighting that, thanks to the third order optimal feedback, the computation of each optimal control law is reduced to the evaluation of a polynomial. Similarly to the lunar landing case, the perturbed solutions are studied in Table 2 in terms of performance index and mass fraction $m_{f} / m_{i}$. More specifically, the table compares their reference values with the minimum and maximum values assumed over all the samples.

The dependence of the accuracy of the polynomial map (21) on the order 


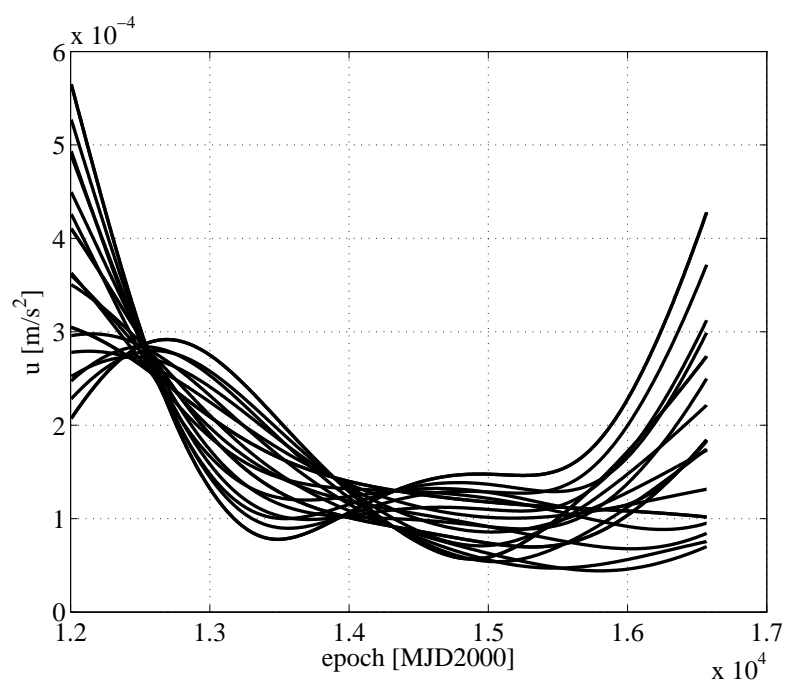

Figure 25: Earth-Mars transfer problem: control magnitude profiles resulting from third order corrections.

Table 2: Earth-Mars transfer problem: performance index and mass fraction range over all samples.

\begin{tabular}{lccc}
\hline Parameter & Nom. value & Min. value & Max. value \\
\hline Performance index $\left[\mathrm{m}^{2} / \mathrm{s}^{3}\right]$ & $1.48390 \cdot 10^{13}$ & $1.43055 \cdot 10^{13}$ & $2.80070 \cdot 10^{13}$ \\
Mass fraction $m_{f} / m_{i}$ & 0.80655 & 0.74538 & 0.81984 \\
\hline
\end{tabular}

is investigated in Fig. 26, Fig. 27, and Fig. 28. More specifically, the imposed square at arrival is compared with the actual final positions obtained with first, second, and third order control corrections, respectively. The figure shows the evident inaccuracy of first order corrections, which are typical of classical linear feedback control algorithms. Higher order corrections drastically reduce the final error. 


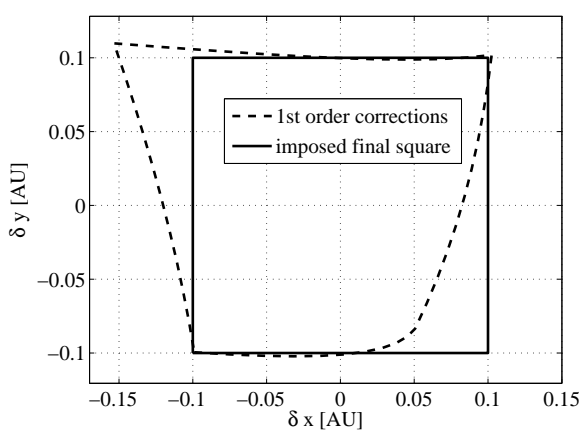

Figure 26: Comparison between imposed square at arrival and actual final positions from first order corrections.

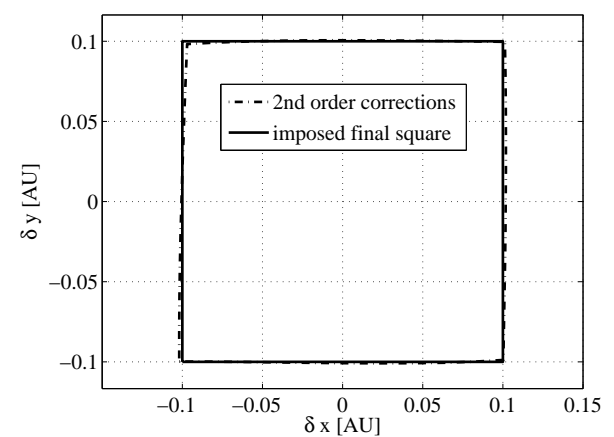

Figure 27: Comparison between imposed square at arrival and actual final positions from second order corrections.

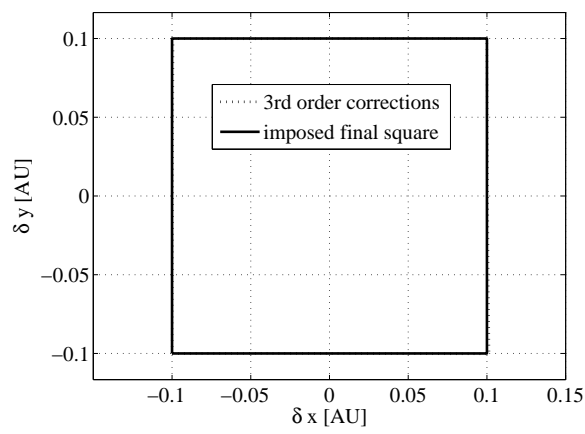

Figure 28: Comparison between imposed square at arrival and actual final positions from third order corrections.

\section{Conclusion}

A method for the computation of optimal feedback control laws based on differential algebra has been introduced, with applications to lunar landing, rendezvous maneuvers, and Earth-Mars transfers. The method relies 
on the high order expansion of the solution of the optimal control problem about a reference trajectory. Thus, it improves the results of classical techniques based on the linearization of the dynamics. Moreover, the method reduces the computation of new optimal control laws to the mere evaluation of polynomials. This is a valuable advantage over the conventional nonlinear optimal control strategies, which are mainly based on iterative procedures. However, the method is not free of limitations. More specifically, it is only applicable to the class of optimal control problems in which the system equations are affine in the control vector. In addition, it can not include control saturation constraints in the version presented in this paper. Finally, this work focused on the problem of transferring a spacecraft from an initial fixed state to a final fixed state, thus omitting the imposition of soft constraints on boundary conditions. Ongoing work is focused to address such limitations.

\section{References}

[1] T. Cimen, Survey of State-Dependent Riccati Equation in Nonlinear Optimal Feedback Control Synthesis, Journal of Guidance, Control, and Dynamics, 35 (2012) 1025-1047.

[2] A. Bryson, Y. Ho, Applied Optimal Control: optimization, estimation, and control, Hemisphere Publishing Co.s, Washigton, 1975.

[3] B. Bonnard, J.B. Caillau, Introduction to nonlinear optimal control, in Advanced topics in control systems theory, Lecture Notes in Control and Inform. Sci., Springer, 2006. 
[4] J.D. Pearson, Approximation methods in optimal control, Journal of Electronics and Control, 13 (1962) 453-469.

[5] A. Wernli, G. Cook, Suboptimal control for the nonlinear quadratic regulator problem, Automatica, 11 (1975) 75-84.

[6] C.P. Mracek, J.R. Cloutier, Control designs for the nonlinear benchmark problem via the state-dependent Riccati equation method, International Journal of Robust and Nonlinear Control, 8 (1998) 401-433.

[7] S.C. Beeler, State-dependent Riccati Equation Regulation of Systems with State and Control Nonlinearities, NIA Report No. 2004-08, National Institute of Aerospace, Hampton, Virginia, 2004.

[8] M. Xin, S.N. Balakrishnan, D.T. Stansbery, E.J. Ohlmeyer, Nonlinear Missile Autopilot Design with $\theta-D$ Technique, Journal of Guidance, Control and Dynamics, 27 (2004) 406-417.

[9] T. Bullock, G. Franklin, A second-order feedback method for optimal control computations, IEEE Transactions on Automatic Control, 12 (1967) 666-673.

[10] J.T. Olympio, Algorithm for low thrust optimal interplanetary transfers with escape and capture phases, AIAA/AAS Astrodynamics Specialist Conference and Exhibit, Honolulu, Hawaii, August 2008.

[11] C. Park, D. Scheeres, Solution of Optimal Feedback Control Problems with General Boundary Conditions Using Hamiltonian Dynamics and Generating Functions, Automatica, 42 (2006), 869-875. 
[12] M. Berz, Modern Map Methods in Particle Beam Physics, Academic Press, New York, 1999.

[13] M. Berz, The new method of TPSA algebra for the description of beam dynamics to high orders, Technical Report No. AT-6:ATN-86-16, Los Alamos National Laboratory, Los Alamos, NM, 1986.

[14] M. Berz, K. Makino, COSY INFINITY version 9 reference manual, MSU Report MSUHEP-060803, Michigan State University, East Lansing, MI, 2006.

[15] J. Stoer, R. Bulirsch, Introduction to Numerical Analysis, Springer, New York, 1993.

[16] P. Di Lizia, Robust Space Trajectory and Space System Design Using Differential Algebra, Ph.D. Dissertation, Politecnico di Milano, Milan, Italy, 2008.

[17] C. Park, V. Guibout, D. Scheeres, Solving Optimal Continuous Thrust Rendezvous Problems with Generating Functions, Journal of Guidance, Control, and Dynamics, 29 (2006) 321-331. 